RELATO DE CASOS

\title{
Câncer Colorretal Sincrônico - Relato de Caso e Revisão de Literatura
}

\section{Sychronous Colorrectal Câncer: A Case Report and Literature Review}

\author{
SÉRGIO MURILO DA SILVA ANDRADE ${ }^{1}$; FÁBIO LIMA PEREIRA² \\ ${ }^{1}$ Médico residente em Cirurgia Geral pelo Hospital Universitário Alcides Carneiro, pertencente à \\ Universidade Federal de Campina Grande; ${ }^{2}$ Médico, coloproctologista e professor da disciplina de Cirurgia Geral da \\ Universidade Federal de Campina Grande - Paraiba - Brasil.
}

\begin{abstract}
ANDRADE SMS; PEREIRA FL. Câncer Colorretal Sincrônico - Relato de Caso e Revisão de Literatura. Rev bras Coloproct, 2007;27(1): 069-079.

RESUMO: Relatamos o caso de uma paciente com tumor maligno em cólon direito e neoplasia sincrônica de reto. Os objetivos deste estudo são discutir as vantagens da colonoscopia na detecção de tumores sincrônicos e avaliar os fatores prognósticos mais relevantes na sobrevida de doentes com câncer colorretal (CCR). Utilizamos como fonte de pesquisa, a coleta de dados em prontuário e busca em base de dados do Medline, Lilacs e Scielo. Concluímos que: 1) A colonoscopia oferece avaliação completa do cólon, com diagnóstico pré-operatório de tumores sincrônicos, o que muitas vezes interfere na conduta terapêutica e 2) Os fatores que mais interferem a sobrevida de pacientes com CCR são o estágio de Dukes, grau de diferenciação do tumor e extensão da ressecção cirúrgica.
\end{abstract}

Descritores: Câncer; colorretal; sincrônico; colonoscopia; pólipos.

\section{INTRODUÇÃO}

Neoplasia maligna mais comum do tubo digestivo, o câncer colorretal (CCR) teve sua incidência aumentada nos últimos anos, principalmente em áreas consideradas de baixo risco (países em desenvolvimento). Vários fatores podem estar envolvidos, como o envelhecimento da população, sedentarismo e hábitos alimentares pouco saudáveis. Estima-se em cerca de meio milhão o número de óbitos por esta doença a cada ano, com tendência a queda na mortalidade nos últimos anos ${ }^{(1)}$.

É a quarta neoplasia maligna mais comum no mundo (superada pelos tumores de pulmão, mama e próstata) e a segunda causa de morte por câncer nos Estados Unidos (2); (3). Nos homens, é o quarto tipo de câncer mais frequiente (depois de pulmão, estômago e próstata) e, no sexo feminino, é superado apenas pelos cânceres de mama e colo de útero. No Brasil, o CCR é a quinta neoplasia maligna mais diagnosticada (ocupando segundo lugar na região Sudeste) e a quarta causa de morte por câncer ${ }^{(4)}$.

São condições hereditárias frequentemente associadas a CCR: a polipose adenomatosa familiar (PAF); o câncer colorretal hereditário sem polipose (HNPCC), também denominada síndrome de Lynch; síndrome do carcinoma colorretal hereditário; polipose juvenil familiar; síndrome do adenoma plano e síndrome de Peutz-Jeghers ${ }^{(5)}$.

Além dos fatores genéticos e ambientais, acredita-se que processos inflamatórios também possam estar envolvidos na gênese do CCR. Nesse sentido, destacam-se as doenças inflamatórias intestinais. $\mathrm{Na}$ doença de Crohn, o risco é menor do que na colite ulcerativa (estimado em mais de $40 \%$ após 25 anos de doença), porém maior do que o da população geral ${ }^{(5)}$. A incidência de CCR esporádico, ou seja, aquele não associado a fatores hereditários, parece aumen-

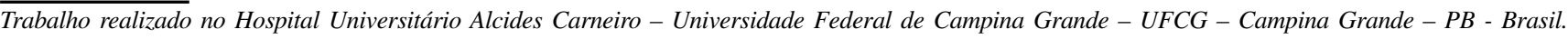


tar com a idade, aparecendo mais frequentemente após a sexta década de vida, sendo que até $90 \%$ de todos os casos ocorrem em pacientes com mais de 50 anos ${ }^{(2) ;}$ ${ }^{(6)}$. A influência da idade no prognóstico, entretanto é controversa na literatura, podendo sofrer influências do estado de saúde do paciente, sendo que muitos estudos relatam maior sobrevida em indivíduos com menos de 40 anos ${ }^{(7) ;(8)}$. O retossigmóide é o sítio primário em cerca de $65 \%$ dos casos em idosos ${ }^{(2)}$.

Até $85 \%$ dos tumores malignos dos cólons ainda são diagnosticados tardiamente, em estádios avançados da doença em pacientes sintomáticos ${ }^{(9)}$. Medidas de rastreio para identificação precoce tumor poderiam diminuir este número, principalmente em pacientes com alto risco, permitindo maiores chances de cura. São considerados indivíduos com risco mais elevado para desenvolvimento de CCR: idade acima de 50 anos, portadores de doença inflamatória intestinal, história pessoal ou familiar de tumores benignos ou malignos de cólon, bem como parente em primeiro grau com CCR sem defeito genético herdado ${ }^{(4) ;(10)}$.

Nos últimos 50 anos, houve um aumento na freqüência de localização do CCR no cólon direito em relação ao cólon esquerdo, sigmóide e reto ${ }^{(5) ;(8)}$. Essa constatação ressalta a importância do uso de métodos diagnósticos que envolvam todo o cólon e não apenas o retossigmóide ${ }^{(5)}$.

Em estudo realizado no Brasil, foram encontradas frequiências de $22,8 \%$ para lesões em cólon direito, $40,6 \%$ para o cólon esquerdo e $36,6 \%$ no reto ${ }^{(8)}$. Em geral, estas frequiências giram em torno de $20 \%$ para o cólon direito, $10 \%$ para o transverso, $10 \%$ em cólon esquerdo, $30 \%$ para sigmóide e outros $30 \%$ para o reto ${ }^{(3)}$.

A realização inicial de biópsia por retossigmoidoscopia pode agilizar o diagnóstico, com 25 a $35 \%$ dos tumores sendo acessíveis para o retossigmoidoscópio rígido ${ }^{(11)}$. No entanto, para uma avaliação mais completa do cólon, com elevada acurácia e inclusive com possibilidade de remoção de pequenos pólipos, o mais indicado é a realização de colonoscopia. São fatores limitantes seu custo mais elevado, necessidade de profissional experiente e tecnologia avançada, com raro risco de perfuração e sangramento ${ }^{(3)}$.

$\mathrm{O}$ aspecto da lesão à retossigmoidoscopia ou colonoscopia é característico, com a borda distal de coloração vinhosa ou avermelhada, podendo tomar as formas ulcerada, anelar ou polipóide. A biópsia da lesão deve incluir tanto o centro como a periferia da lesão ${ }^{(9)}$.
Exames laboratoriais podem ser importantes para avaliação das condições clínicas do paciente, como o hemograma para quantificação da anemia devida a sangramento intestinal crônico, ou no seguimento. Neste caso, são utilizados mais freqüentemente marcadores tumorais como o antígeno carcinoembrionário (CEA) e o CA 19-9, que são, na verdade, pouco específicos, alterando-se também em doenças inflamatórias, alcoolismo e tabagismo, existindo também tumores que não expressam antígenos. Logo, não servem para diagnóstico e sim para seguimento do paciente, com uma dosagem inicial na avaliação pré-operatória e mensurações periódicas no pós-tratamento. Enzimas hepáticas também são úteis na pesquisa de metástases para o fígado ${ }^{(9)}$.

O enema opaco simples foi, por anos, o único meio diagnóstico para tumores não alcançados pelo toque retal ou pela retossigmoidoscopia rígida até que, em 1967, Welin descreveu o duplo contraste, com bário e $\operatorname{ar}^{(9)}$.

Introduzida como exame auxiliar de grande importância no diagnóstico do CCR em 1967, a colonoscopia é considerada por muitos autores complementar ao enema opaco, tendo a grande vantagem em relação a este de permitir biópsia da lesão suspeita e estadiamento.

Pacientes com CCR, mesmo os de localização distal, precisam ser avaliados em todo o cólon (através de colonoscopia), pois, além de ter havido um aumento na incidência de tumores à direita em relação ao retossigmóide, existe a possibilidade de tumores sincrônicos ${ }^{(5) ;(9)}$. Tumores são ditos sincrônicos quando se apresentam simultaneamente ao diagnóstico ou no ato operatório, descartando-se obviamente a possibilidade de lesões por contigüidade. Tumores metacrônicos são encontrados no seguimento dos pacientes já submetidos à ressecção cirúrgica, excluindo-se evidentemente a possibilidade de lesão não diagnosticada à época do diagnóstico (quando seria sincrônica) ou de recidiva do tumor operado. Pólipos sincrônicos ocorrem em até 18 a $27 \%$ dos casos. No caso de tumores malignos, essa frequiência é de 1,5 até $8 \%{ }^{(9) ;(12)}$. Neoplasias metacrônicas são encontradas no seguimento de até 0,6 a $8 \%$ dos pacientes ${ }^{(13)}$.

Os avanços em termos de diagnóstico do CCR incluem ainda a ultra-sonografia endoscópica, cromoscopia e a colonoscopia virtual, com recursos de tomografia computadorizada, simulando uma colonoscopia verdadeira, com imagens tridimensionais. São ferramentas importantes inclusive para imediato 
estadiamento, mas seu custo elevado não permite sua realização na maioria dos centros diagnósticos ${ }^{(2) ;(3)}$.

O CCR dissemina-se por contigüidade (invasão de tecidos vizinhos), implante no peritônio ou órgãos intra-abdominais e pelas vias linfática e hematogênica. Os principais órgãos envolvidos em metástases são o fígado (75\%), pulmões (15\%), ossos $(5 \%)$ e sistema nervoso central (5\%). O adenocarcinoma é o tipo histológico mais freqüente, encontrado em até $90 \%$ dos casos ${ }^{(3)}$.

O estadiamento tem implicações prognósticas , sendo as classificações mais utilizadas a de DUKES modificada em 1974 (por Gunderson e Sosin) e o sistema TNM, podendo-se usar também a de Astler e Coller (quadros 1, 2 e 3).

O tratamento do CCR ainda é eminentemente cirúrgico, com as terapias adjuvantes pouco alterando a mortalidade e contribuindo para melhora da qualidade de vida. A sobrevida global em cinco anos de pacientes submetidos a tratamento cirúrgico gira em torno de $50 \%{ }^{(3)}$. Há intenção curativa quando se promove a remoção completa do tumor primário, órgãos ou estruturas adjacentes comprometidos, com margem circunferencial e radial histologicamente livres (ressecção em bloco). Tratamento radio e quimioterápicos podem apresentar vantagens nos estádios II e III ${ }^{(3) ;(14)}$.Os quadros 4 e 5 apresentam as diretrizes do National Cancer Institute - U.S. National Institutes of Health para tratamento do CCR.

O prognóstico do CCR está intimamente relacionado ao estádio da doença, chegando a $90 \%$ de chance de cura no estádio I, correspondente à categoria A de Dukes, e sendo menor que 5\% para pacientes com tumores em estádio IV, ou categoria D de
Dukes ${ }^{(6)}$. No seguimento de tais pacientes, a colonoscopia seriada é imprescindível, dada a frequiência variável de adenomas e tumores sincrônicos e metacrônicos.

Este artigo se propõe a mostrar um relato de caso de adenocarcinomas sincrônicos de ceco e reto, uma ocorrência rara em se tratando de câncer colorretal. Serão discutidas as vantagens da colonoscopia em relação a métodos pouco invasivos de diagnóstico na detecção de tumores sincrônicos e aferidas as principais variáveis prognósticas do CCR para seguimento em longo prazo e risco de recorrência (em termos de recidiva tumoral ou aparecimento de lesões metacrônicas).

\section{RELATO DO CASO}

Paciente I.B.S., sexo feminino, branca, 64 anos, natural de Areia - PB e procedente de Remígio - PB, casada, aposentada e ex-cozinheira. Queixa de dor abdominal, em fossa ilíaca direita, de forte intensidade, contínua, em pontada, iniciada dois meses antes da consulta. Acompanhava mudança de hábito intestinal, que passou de evacuações diárias com fezes bem formadas a eliminações diarréicas (cerca de seis vezes ao dia) com muco e melena. Houve ainda crescimento de tumoração em topografia de fossa ilíaca direita, anorexia e perda de peso de cerca de $6 \mathrm{~kg}$ no período.

Nos antecedentes pessoais encontramos relato de menopausa aos 55 anos, gesta VIII, para VII. Passado cirúrgico de colecistectomia há 3 anos e histerectomia total há 5 anos por tumor de endométrio, que se revelou à histologia como um adenocarcinoma pouco diferenciado. Realizou, além do tratamento cirúrgico, sessões de radioterapia e braquiterapia.

\section{Quadro 1 - Estadiamento patológico de Dukes modificado por Gunderson e Sosin (1974).}

\begin{tabular}{|ll|}
\hline A & Tumor limitado à mucosa, linfonodo negativo \\
B1 & Tumor além da mucosa, mas limitado à parede intestinal, linfonodo negativo \\
B2 & Tumor além da parede intestinal, linfonodo negativo; \\
& $B 2 m$ : extensão microscópica \\
B3 & Tumor aderindo ou invadindo órgãos adjacentes, linfonodo positivo \\
C1 & B1 com linfonodo positivo \\
C2 & B2 com linfonodo positivo; \\
& $C 2 m: B 2 m$ com linfonodo positivo \\
C3 & B3 com linfonodo positivo \\
D & Metástase à distância \\
\hline
\end{tabular}


Quadro 2 - Classificação TNM.

\section{Tumor primário $(\mathrm{T})$}

TX Tumor primário não pode ser avaliado

T0 Sem evidência de tumor primário

Tis Carcinoma in situ: intra-epitelial ou invasão da lâmina própria*

T1 Tumor invade a submucosa

T2 Tumor invade a muscular própria

T3 Tumor invade além da muscular própria, sem ultrapassar a subserosa ou os tecidos desperitonizados pericólicos ou perirretais

T4 Tumor invade diretamente outros órgãos ou estruturas e/ou perfura o peritônio visceral ${ }^{* *} * * *$

$$
\text { Linfonodos regionais }(\mathrm{N})
$$

NX N não pode ser avaliado

N0 Ausência de metástase linfonodal

N1 Metástase para $1-3$ linfonodos

N2 Metástase para mais de 4 linfonodos

Metástase (M)

MX M não pode ser avaliada

M0 Ausência de metástase

M1 Presença de metástase

Alérgica ao piroxicam. Nega tabagismo e etilismo. Relato de dieta balanceada, com três refeições diárias. Conhece o triatomíneo e refere banhos de rio na infância. Nega diabetes melito e hipertensão arterial.
Ao exame físico apresentava-se em estado geral regular, emagrecida e normocorada. Ausculta cardiovascular normal. Abdome normotenso e com massa palpável em fossa ilíaca direita, dolorosa. Si-

Quadro 3 - Estadiamento TNM.

\begin{tabular}{|lccccc|}
\hline & & \multicolumn{2}{c|}{ Estádios } & & \\
Estádio & $\mathrm{T}$ & $\mathrm{N}$ & $\mathrm{M}$ & Dukes & MAC \\
0 & Tis & $\mathrm{N} 0$ & $\mathrm{M} 0$ & - & - \\
I & T1 & $\mathrm{N} 0$ & $\mathrm{M} 0$ & $\mathrm{~A}$ & $\mathrm{~A}$ \\
& $\mathrm{~T} 2$ & $\mathrm{~N} 0$ & $\mathrm{M} 0$ & $\mathrm{~A}$ & $\mathrm{~B} 1$ \\
IIA & $\mathrm{T} 3$ & $\mathrm{~N} 0$ & $\mathrm{M} 0$ & $\mathrm{~B}$ & $\mathrm{~B} 2$ \\
IIB & $\mathrm{T} 4$ & $\mathrm{~N} 0$ & $\mathrm{M} 0$ & $\mathrm{~B}$ & $\mathrm{~B} 3$ \\
IIIA & $\mathrm{T} 1-\mathrm{T} 2$ & $\mathrm{~N} 1$ & $\mathrm{C} 0$ & $\mathrm{C}$ & $\mathrm{C} 1$ \\
IIIB & T3 $-\mathrm{T} 4$ & $\mathrm{~N} 1$ & $\mathrm{C} 0$ & $\mathrm{C} 2 / \mathrm{C} 3$ \\
IIIC & Qualquer T & $\mathrm{N} 2$ & $\mathrm{C} 0$ & $\mathrm{C} 1 / \mathrm{C} 2 / \mathrm{C} 3$ \\
IV & Qualquer T & Qualquer N & M1 & $\mathrm{D}$ & $\mathrm{D}$ \\
\hline
\end{tabular}

* Tis inclui tumores confinados dentro da membrana basal glandular (intra-epitelial) ou lâmina própria (intramucoso)sem extensão através da muscular da mucosa.

** Invasão direta em T4 inclui invasão de outros segmentos colorretais através da serosa, por exemplo: invasão do cólon sigmóide por um carcinoma de ceco.

*** Tumor aderido a outros órgãos ou estruturas é classificado como T4 se a análise histopatológica confirmar aderência neoplásica. As letras Ve L devem ser utilizadas para identificar a presença de invasão vascular e linfática, respectivamente.

Nota: Os prefixos y e r devem ser utilizados para o estadiamento após tratamento neo-adjuvante e recidiva, respectivamente. 
Quadro 4 - Tratamento do câncer de cólon.

\begin{tabular}{|ll|}
\hline Estágio 0: Tis, N0, M0 & $\begin{array}{l}\text { Excisão local ou polipectomia } \\
\text { Ressecção colônica se necessário } \\
\text { Estágio I: T1, N0, M0; T2, N0, M0 }\end{array}$ \\
Estágio IIA: T3, N0, M0 & Ressecção colônica \\
Estágio IIB: T4, N0, M0 & Ressecção colônica + quimioterapia \\
Estágio IIIA: T1, N1, M0; T2, N1, M0 & \\
Estágio IIIB: T3, N1, M0; T4, N1, M0 & \\
Estágio IIIC: qualquer T, N2, M0 & Ressecção colônica + quimioterapia; \\
Estágio IV: qualquer T ou N, M1 & Resseção de metástases isoladas \\
\end{tabular}

* Preferentemente em ensaios clínicos controlados.

nais de insuficiência venosa periférica em membros inferiores.

O exame proctológico revelava inspeção normal e palpação sem anormalidades. Ao toque retal, com esfíncter normotônico, evidenciava-se a presença de lesão vegetante em QAD iniciando-se na linha pectínea e se extendendo até cerca de $3 \mathrm{~cm}$ acima. A anuscopia confirmou lesão em QAD, não biopsiada devido à biópsia prévia por colonoscopia trazida pela paciente.

Submetida ao exame ultra-sonográfico em maio de 2005, foi evidenciado massa sólida heterogênea, medindo aproximadamente $8,0 \times 7,0 \times 7,6 \mathrm{~cm}$, localizada em topografia de fossa ilíaca direita, podendo ser de origem intestinal ou ovariana, com fígado, rins, pâncreas e baço sem alterações.

A colonoscopia, trazida pela paciente, revelava uma lesão vegetante e estenosante, friável e sangrante, localizada provavelmente em ângulo hepá- tico do cólon / cólon ascendente, além de divertículos hipotônicos de cólon transverso, polipose dos cólons, angiodisplasia de ângulo esplênico e pólipo Yamada III de canal anal (reto baixo), sendo biopsiados o tumor do cólon e o pólipo do reto baixo.

O histopatológico do tumor colônico não foi conclusivo, enquanto o pólipo do canal anal (reto baixo) evidenciava adenocarcinoma bem diferenciado infiltrante.

O hemograma pré-operatório (junho de 2005) não mostrava anemia nem sinais de infecção. $O$ coagulograma e a bioquímica (glicemia de jejum, uréia, creatinina, proteínas totais e albumina) também estavam normais.

Os achados intra-operatórios foram de um volumoso tumor de ceco, gânglios para-aórticos livres e ausência de implantes peritoneais ou em fígado. O tumor invadia até a serosa, com infiltração de íleo termi-

Quadro 5 - Tratamento do câncer de reto.

Estágio 0: Tis, N0, M0
Estágio I: T1, N0, M0; T2, N0, M0
Estágio IIA: T3, N0, M0
Estágio IIB: T4, N0, M0
Estágio IIIA: T1, N1, M0; T2, N1, M0
Estágio IIIB: T3, N1, M0; T4, N1, M0
Estágio IIIC: qualquer T, N2, M0
Estágio IV: qualquer T ou N, M1

* Preferentemente em ensaios clínicos controlados
Excisão local ou polipectomia

Ressecção da parede retal se necessário

Radioterapia*

Retossigmoidectomia anterior +

Radioterapia + quimioterapia*

Retossigmoidectomia anterior em bloco +

Radioterapia + quimioterapia*

Retossigmoidectomia anterior em bloco +

Radioterapia + quimioterapia*

Retossigmoidectomia anterior + ressecção de metástases isoladas + radioterapia + quimioterapia 
nal. Havia também tumoração em reto baixo, logo acima da linha pectínea (ver figuras 1 e 2).

A cirurgia com intenção curativa foi uma proctocolectomia total com ileostomia a Brooke em fossa ilíaca direita (ver figuras), sendo enviado material para análise histopatológica.

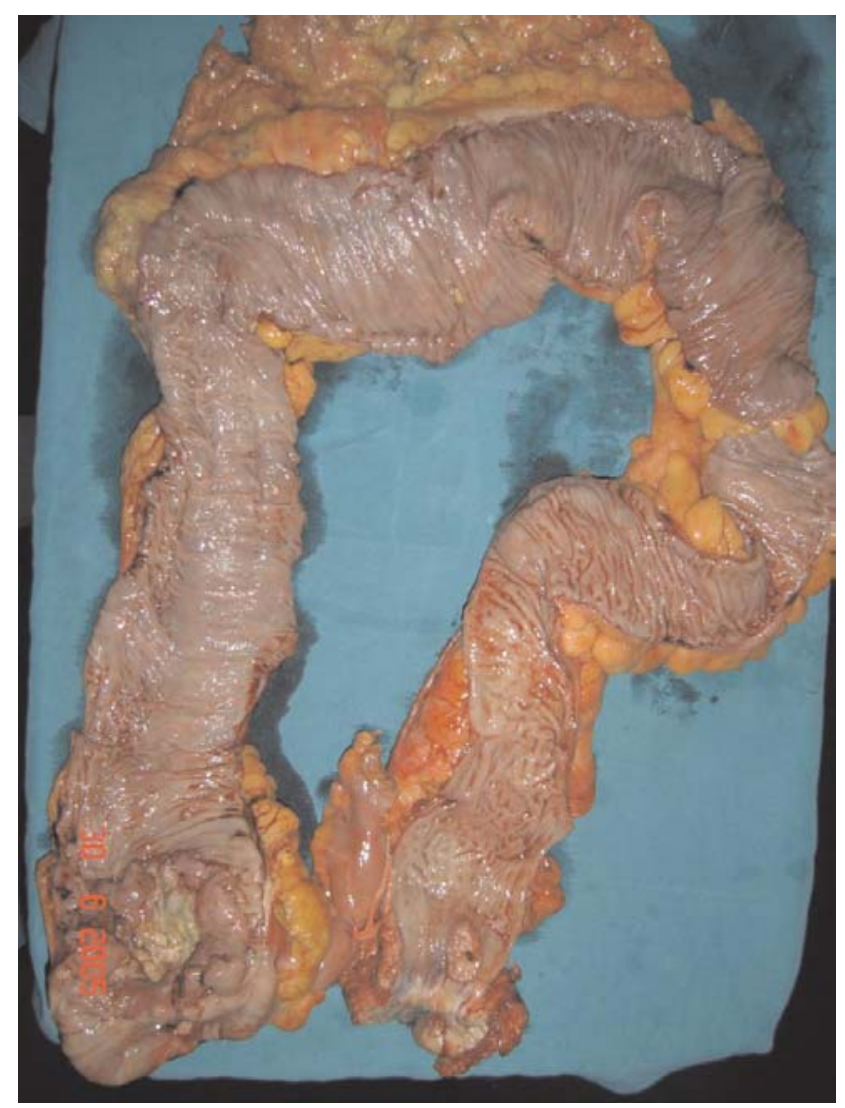

Figura 1 - Peça cirúrgica aberta, evidenciando os tumores de ceco (à esquerda) e reto baixo (à direita).

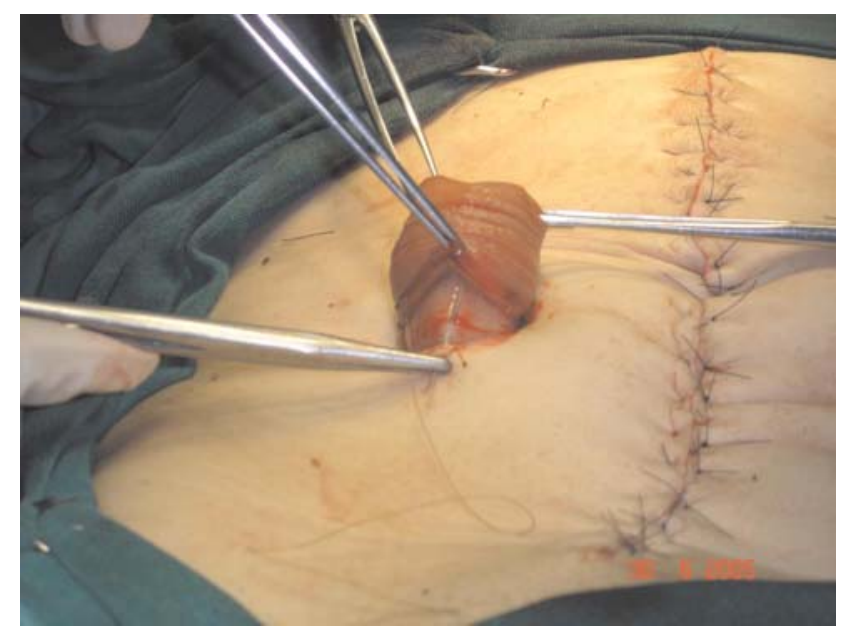

Figura 2 - Ileostomia a Brooke.
O exame anátomo-patológico revelou uma neoplasia localizada no ceco, medindo $5,0 \times 4,0 \mathrm{~cm}$, ulcero-infiltrativa, com perfuração macroscópica infiltrando tecidos moles pericólicos, e outra situada no reto, elevada, tipo placa, medindo $2,5 \times 1,4 \mathrm{~cm}$, com infiltração até a muscular própria e distando $3,6 \mathrm{~cm}$ da margem de ressecção cirúrgica (margem anal). Havia ainda, metástases para 2 linfonodos pericólicos de 10 isolados. Mucosa intestinal adjacente sem alterações histológicas significativas. Invasão neoplásica em parede de íleo terminal. Tratava-se de adenocarcinomas moderadamente diferenciados.

O estadiamento TNM para o tumor de ceco, portanto, foi T4N1M0 (estágio IIIB) ou C de Dukes. Para o tumor de reto, o estágio foi IIA (T3NOM0) ou B de Dukes.

O seguimento está sendo realizado a cada 3 meses com exames clínicos de rotina, solicitação do antígeno carcinoembrionário (CEA), hemograma e exames radiológicos de tórax e ultra-sonográficos de abdome a cada 6 meses. A paciente encontra-se ainda em protocolo de quimioterapia e acompanhamento com a oncologia.

\section{MÉTODO}

Foi realizada coleta de dados em prontuário da paciente durante seu acompanhamento na Fundação Assistencial da Paraíba (FAP), de maio a julho de 2005, correspondendo ao período pré e pós-operatório imediato, após assinatura do termo de consentimento específico.

A revisão de literatura específica utilizou a base de dados do Medline, Lilacs, Scielo e Pubmed, além de consulta a periódicos e livros-texto na área.

Este trabalho obteve a aprovação do Comitê de Ética em Pesquisa do Hospital Universitário Alcides Carneiro - Campina Grande - PB.

\section{DISCUSSÃO}

\subsection{Uso do método colonoscópico no di- agnóstico do CCR}

Introduzida em 1967 como exame complementar, a colonoscopia assumiu grande papel no diagnóstico das afecções colônicas, notadamente do câncer colorretal (CCR), possibilitando uma visão macroscópica em tempo real do cólon em toda a sua extensão. 
Até então, o enema opaco e a retossigmoidoscopia rígida eram os recursos mais utilizados para identificação de lesões suspeitas de CCR. Um complementava o outro, pois enquanto o primeiro mostrava todo o cólon, o segundo permitia uma visualização macroscópica, porquanto fosse segmentar, englobando apenas a porção distal do cólon (sigmóide) e reto. Assim, uma vez identificada lesão suspeita à retossigmoidoscopia rígida, a realização do enema opaco ajudava na busca por lesões sincrônicas, sugerindo algumas vezes a retirada de determinadas imagens suspeitas.

A ultra-sonografia e a tomografia computadorizada tiveram sempre valor limitado para identificação de doença nos cólons, servindo mais para auxiliar no estadiamento, detectando acometimento de órgãos ou estruturas vizinhas.

Algumas lesões, entretanto, poderiam passar despercebidas ao exame contrastado, como pequenos pólipos. A colonoscopia surge então com inúmeras vantagens, como a visualização endoscópica de todo o cólon e também do íleo terminal, identificação direta do tumor, realização de biópsia e o diagnóstico e ressecção de pólipos, bloqueando uma possível progressão para carcinoma, tornando-se mandatória sua realização no pré-operatório ${ }^{(2) ;(15) ;(16) .}$

O uso de técnicas de ultra-sonografia e de tomografia computadorizada acopladas à colonoscopia permite ainda auxiliar no estadiamento, com imagens tridimensionais, quantificando a invasão tecidual do tumor, bem como o acometimento de estruturas vizinhas (2); (3).

Uma das mais importantes contribuições da colonoscopia para o diagnóstico do CCR é, sem dúvida, a identificação de lesões sincrônicas, sejam elas benignas (pólipos adenomatosos) ou malignas, como foi demonstrado neste relato de caso. Como definido anteriormente, tumores são ditos sincrônicos quando se apresentam simultaneamente ao diagnóstico ou no ato operatório, descartando-se obviamente a possibilidade de lesões por contigüidade ${ }^{(12)}$. O CCR esporádico pode associar-se à presença de outros tumores malignos em 1,5 a $8 \%$ (em média 4\%) dos casos e de pólipos adenomatosos sincrônicos numa frequiência de cerca de $25 \%$, variando em geral de 18 a $27 \%$ dos casos (2); (9); (12). Variáveis como sexo masculino e a presença de pólipos à colonoscopia, já foram citadas como sinal de alerta para a possível existência de neoplasia maligna sincrônica ${ }^{(17)}$. Lesões neoplásicas sincrônicas não identificadas no pré ou intra-operatório podem levar a tratamento cirúrgico inadequado, implicando em segunda intervenção com o tumor muitas vezes em estádio avançado, sem possibilidade de tratamento curativo ${ }^{(12)}$. Os conceitos de sincronismo e metacronismo estão associados à hipótese de que todo o cólon está exposto ao fator (ou fatores) de risco que desencadearam o CCR, havendo, portanto, a possibilidade de coexistência de lesões em diferentes estágios de desenvolvimento tumoral no intestino grosso.

O caso clínico em questão é bastante ilustrativo do papel da colonoscopia na identificação de lesões sincrônicas, que podem inclusive modificar a proposta terapêutica. Em se confirmando a presença de neoplasias sincrônicas, preconiza-se a realização de colectomia total ou subtotal, desde que as condições clínicas do paciente permitam ${ }^{(12)}$. A paciente em estudo foi submetida a uma proctocolectomia total com ileostomia, justamente porque apresentava concomitantemente lesões malignas no reto inferior e cólon ascendente, além da presença de polipose dos cólons. A lesão em canal anal(reto baixo) apresentava-se sob a forma de pólipo que, submetido a biópsia e exame histopatológico, revelou-se um adenocarcinoma bem diferenciado e infiltrante. $\mathrm{O}$ enema baritado não foi realizado, porém, mesmo que detectasse pólipo suspeito ou mesmo a polipose dos cólons, a biópsia só seria possível através de visualização endoscópica.

$\mathrm{O}$ aumento na frequiência de lesões malignas em cólon direito em relação ao retossigmóide ressalta a importância da utilização de métodos diagnósticos que envolvam todo o cólon ${ }^{(5) ;(8) ;(18)}$. Estudo conduzido aqui no Brasil comparando as vantagens da realização de colonoscopia em relação à retossigmoidoscopia isolada revelou que $22,77 \%$ dos pacientes possuíam lesões isoladas (adenomas ou adenocarcinomas) em cólon direito ${ }^{(19)}$. Outro estudo com 28 pacientes portadores de adenocarcinoma colorretal sincrônicos revelou que $32 \%$ das lesões encontravam-se em cólon direito, 32\% em cólon esquerdo, $26 \%$ no reto e $10 \%$ no cólon transverso ${ }^{(12)}$.

A paciente em questão apresentava lesões malignas sincrônicas em reto inferior e cólon direito, mais precisamente em ceco. Como o diagnóstico foi tardio, a lesão à direita tomou tal proporção, que foi identificada por exame ultra-sonográfico de abdome. Provavelmente, em fase mais precoce de sua doença ela possuísse apenas a neoplasia isolada de cólon direito e uma colonoscopia de rastreio realizada após os 50 
anos, subsidiada por uma pesquisa de sangue oculto positiva como triagem, revelasse a lesão, possibilitando talvez um tratamento cirúrgico menos agressivo e com maior chance de cura.

Quanto à sensibilidade da colonoscopia em relação ao exame contrastado, encontramos que é necessária a sua realização após cerca de $40 \%$ dos enemas opacos, por preparo inadequado dos cólons (o que também dificulta a realização da colonoscopia) ou dúvidas em relação àquele exame. Pode haver, no entanto, similaridade em termos de sensibilidade entre o exame endoscópico dos cólons e o duplo contraste para a identificação de CCR em pacientes assintomáticos (2). Estudo conduzido na Argentina encontrou $42,8 \%$ de sensibilidade para a radiologia contrastada, $78,6 \%$ em se tratando de colonoscopia e $97 \%$ para colonoscopia associada à palpação intraoperatória ${ }^{(17)}$.

Não podemos fazer uma comparação entre os achados endoscópicos e radiológicos contrastados em nosso caso clínico, pois a paciente não realizou enema opaco com duplo contraste.

Diminuição de sensibilidade da colonoscopia pode ocorrer em casos de preparo inadequado dos cólons (como na doença diverticular) ou em locais com dificuldade de acesso, como a região por trás das flexuras colônicas, por exemplo, quando completa visualização do cólon não é possível ${ }^{(2)}$.

No seguimento de pacientes no pós-operatório de CCR, a colonoscopia também assume relevância, pela frequiência variável de lesões metacrônicas ou mesmo de recidiva tumoral. A maioria das recorrências, que aparecem em cerca de $50 \%$ dos pacientes com margens cirúrgicas macroscopicamente livres de doença, ocorrem nos primeiros 24 meses após a cirurgia (3). Foi encontrada uma frequiência de pólipos adenomatosos de $18 \%$ no seguimento de pacientes submetidos à ressecção de CCR, maior no cólon ascendente (já que o cólon esquerdo e retossigmóide em geral são ressecados) e nos primeiros dois anos de acompanhamento ${ }^{(20)}$.

Assim, preconiza-se a realização de colonoscopia de 12 a 15 meses após a cirurgia de ressecção do tumor primário e, no seguimento, pelo menos a cada 24 meses, para detecção precoce de recidivas ou de tumores metacrônicos ${ }^{(21)}$. A Sociedade Americana de Câncer, Colégio Americano de Gastrenterologia e a Sociedade Americana de Cirugiões Colorretais recomendam uma periodicidade de 3 a 5 anos para a realização de colonoscopia no seguimento de pacientes com CCR ${ }^{(2)}$.

No caso específico de nossa paciente, a realização de acompanhamento colonoscópico é desnecessária, uma vez que ela foi submetida a proctocolectomia com ileostomia. Seu seguimento é feito a cada 3 meses com dosagem de antígeno carcinoembrionário (CEA) sérico, além de radiologia do tórax e ultrasonografia abdominal para pesquisa de metástases e hemograma e bioquímica de rotina.

\subsection{Fatores prognósticos no CCR}

São considerados fatores independentes afetando a sobrevida de pacientes com CCR, a extensão da ressecção, o grau de diferenciação do tumor, a presença e número de linfonodos comprometidos e o

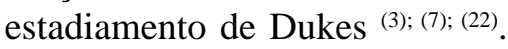

\subsubsection{Idade e sexo}

Alguns autores atribuem maior sobrevida a pacientes mais jovens, provavelmente pela maior freqüiência de doença localizada nestes casos ${ }^{(1)}$. No entanto, há grande discordância na literatura sobre a influência da idade no prognóstico, com muitos autores acreditando que, na verdade, são as condições clínicas do paciente que alteram a mortalidade ${ }^{(7) ;(8)}$. Sobrevida de 5 anos em idosos gira em torno de 22,4 a $56 \%{ }^{(22) ;}$ (23). FARHOUD (2002) encontrou sobrevida de 5 anos em $72,7 \%$ dos pacientes com menos de 40 anos e de $62,3 \%$ na população entre 40 e 60 anos ${ }^{(8)}$. Pacientes com CCR antes dos 40 anos, mais comumente são portadores de síndrome genética familiar com elevada penetrância ${ }^{(1)}$.

A idade isoladamente, não foi fator de risco para mortalidade pós-operatória ou de sobrevida a longo-prazo em pacientes com mais de 75 anos. Fatores mais relacionados à mortalidade foram a perda ponderal, estádio de Dukes, extensão da ressecção e tipo de cirurgia ${ }^{(22)}$. Variáveis fisiológicas, condições clínicas e estadiamento parecem contribuir mais que a idade na decisão de tratamento curativo ou conservador.

Quanto ao sexo, os maiores índices de sobrevivência têm sido relatados no gênero feminino, sendo que mulheres que nunca engravidaram parecem ter sobrevida semelhante à dos homens ${ }^{(8)}$. Foram implicadas não somente causas hormonais como também anatômicas (pelve mais estreita no homem, pro- 
movendo maior relação com órgãos vizinhos e dificultando a extirpação do tumor) para explicar essa proteção em relação ao sexo feminino ${ }^{(8)}$.

\section{croscópicas \\ 4.2.2. Estadiamento e variáveis macro e mi-}

Grande parte dos pacientes são diagnosticados em fases avançadas da doença, o que empobrece o prognóstico, pois as chances de cura despencam em até $90 \%$ nos casos de estádio I ou categoria A de Dukes para menos de $5 \%$ em doentes em estádio IV ou categoria D de Dukes ${ }^{(5)}$. O estadiamento no momento do diagnóstico é considerado por muitos autores o melhor indicador prognóstico ${ }^{(6)}$.

O tamanho do tumor ao diagnóstico, entretanto, parece não influenciar no prognóstico para maioria dos estudos, fato relevante quando se considera a importância que a classificação TNM atribui a esta característica ${ }^{(8)}$.

Variáveis microscópicas, como grau de diferenciação e tipo histológico do tumor, são de grande relevância prognóstica. Lesões bem diferenciadas cursam em geral com maiores taxas de sobrevida ${ }^{(3) ;(7)}$. $\mathrm{O}$ adenocarcinoma mucinoso, em qualquer grau de diferenciação, é considerado fator localmente agressivo e de pior prognóstico ${ }^{(6)}$. Quanto ao aspecto macroscópico, formações vegetantes ou exofíticas aumentam a sobrevida, provavelmente por evoluírem com menores taxas de metástases à distância e recidivas ${ }^{(8)}$.

A paciente em estudo possuía adenocarcinomas moderadamente diferenciados e com estágio $\mathrm{C}$ de Dukes para a neoplasia de ceco. É relatada uma sobrevida de 5 anos em 30 a $40 \%$ para pacientes neste estágio ${ }^{(3)}$. O fato de ser lesão moderadamente diferenciada e do tipo ulcero-infiltrativo ao invés de vegetante empobrece ainda mais esse prognóstico.

No entanto, a ressecção neste caso é considerada completa, pois não havia margens comprometidas. A extensão da ressecção é considerada variável independente, interferindo na sobrevida (3); (22). Uma ressecção completa, portanto curativa, é indicativa de melhor prognóstico.

\subsubsection{Localização do tumor}

No Brasil, um estudo identificou frequiência de $22,8 \%$ de lesões situadas em cólon direito, $40,6 \%$ em cólon esquerdo e $36,6 \%$ no reto, sendo notável nos últimos anos um decréscimo para as neoplasias retais, enquanto cresce a taxa de tumores em cólon direito ${ }^{(8)}$.

Há muitas controvérsias na literatura acerca do valor prognóstico do sítio da neoplasia ${ }^{(8)}$. Muitos estudos revelam maiores taxas de sobrevida para tumores em cólon direito, com piora do prognóstico à medida que as lesões se situam mais distalmente (6); ${ }^{(24)}$. Entretanto, algumas casuísticas revelam que a melhor sobrevida ocorre para tumores em cólon esquerdo $(63,9 \%)$, seguida pelas lesões em cólon direito e reto $\left(58,9\right.$ e $51,3 \%$, respectivamente) ${ }^{(8)}$. As lesões retais em geral são as de pior prognóstico, com taxas de sobrevida que variam de 62 a $66 \%$, em detrimento de tumores colônicos, onde essas taxas encontram-se entre 74 e 77,5\% em estudos mais recentes ${ }^{(8)}$.

A lesão da paciente em questão em cólon direito poderia sugerir um prognóstico melhor, mas o tumor sincrônico retal é o que apresenta os piores índices de sobrevida.

\subsubsection{Relação com outros tipos de câncer}

Este é um fator que poderia ser extraído deste relato de caso específico, pois, na história clínica da paciente, identificamos um passado cirúrgico de histerectomia e tratamento radioterápico por tumor maligno de endométrio. É reconhecida a relação existente entre CCR e a maior incidência de outros tipos de adenocarcinoma, como o de mama, ovário, estômago e também de endométrio, característica da síndrome de Lynch II (um tipo de carcinoma colorretal hereditário não-polipóide), de herança autossômica dominante, predominância de tumores em cólon proximal e maior risco para lesões sincrônicas e metacrônicas ${ }^{(5)}$. A média de idade para o diagnóstico, no entanto, é precoce (em média 44 anos), com a paciente em questão apresentando 59 anos à época do primeiro tumor (endométrio) e 64 anos para o CCR.

Mas o fato de a paciente ter história de adenocarcinoma de endométrio, tumor em cólon proximal (ceco) e lesões sincrônicas deve alertar para o aparecimento de outros tipos de tumor, exigindo seguimento mais rigoroso, com atenção para os sítios mais frequentemente envolvidos na síndrome de Lynch II. Ainda, parentes em primeiro grau constituem grupo de risco aumentado para CCR, devendo ser submetidos a medidas de rastreio para identificação precoce de tumor. 
Rev bras Coloproct

Janeiro/Março, 2007
Câncer Colorretal Sincrônico - Relato de Caso e
Revisão de Literatura
Sergio Murilo da Silva Andrade e Cols.

e a extensão da ressecção cirúrgica. A idade não é fator isolado relevante, dependendo muito mais de variáveis clínicas.

A paciente em questão tem como indicativo de bom prognóstico a ressecção, considerada completa e com margem cirúrgica, mas o estágio de Dukes avançado do tumor de ceco e seu grau de diferenciação moderado são fatores que influenciam negativamente sua sobrevida em longo prazo.

\begin{abstract}
That is a case report of a patient with a cancer of right colon and synchronous neoplasia of rectum. The objectives of this study are to discuss advantages of colonoscopy in diagnosis of synchronous tumor and to discern about more relevant prognostic factors in long-term survival of patients with colorectal cancer (CRC). Our sources of research were patient's records and Medline, Lilacs and Scielo data base. We conclude that: 1) Colonoscopy allows complete evaluation of colon with preoperative diagnose of synchronous tumors, what is important to surgical treatment most of all, and 2) The most important factors in survival of patients with CRC are Dukes' stage, degree of differentiation of tumor and extension of surgery resection.
\end{abstract}

Key words: Cancer; colorrectal; synchronous; colonoscopy; polyp.

\section{REFERÊNCIAS}

1. DUARTE-FRANCO, E; FRANCO, EL. Epidemiologia e fatores de risco em câncer colorretal. In: ROSSI, BM; et al. Câncer de cólon, reto e ânus. $1^{a}$. Ed. São Paulo: Lemar e Tecmedd Editora, 2004. p.3 - 21.

2. ARAUJO SEA et al. - Papel da colonoscopia no câncer colorretal. Rev. Hosp. Clín. Fac. Med. S. Paulo 56(1):25-35, 2001.

3. CHIELE NETO, C; TARTA, C. Câncer colorretal. In: ROHDE, $\mathrm{L}$; et al. Rotinas em cirurgia digestiva. $1^{\mathrm{a}}$. Ed. Porto Alegre: Artmed, 2005. p. 187 - 92.

4. FANG CHIA, BIN. Rastreamento para câncer colorretal. Rev. Assoc. Med. Bras., Dez 2002, vol.48, no.4, p.286-286.

5. FRY, RD. Cólon, reto e ânus. In: JARRELL, BE; CARABASI, RA. National medical series - cirurgia. $3^{\text {a }}$. Ed. Rio de Janeiro: Guanabara Koogan, 2001. p. 215 - 47.

6. MONTEIRO, EP; et al. Neoplasia colorretal até 40 anos: experiência em cinco anos. Rev bras. colo-proctol., Jun 2006, vol.26, no.2, p.156-161.

7. DEANS GT, HEATLEY M, PATTERSON CC, Moorehead RJ, Parks TG, Rowlands BJ, Spence RAJ. Colorectal carcinoma: importance of clinical and pathological factors in survival. Ann R Coll Surg Engl 1994;76:59-64.

8. FARHOUD, S; et al. Variáveis clínicas e macroscópicas que influenciam o prognóstico do carcinoma colorretal. Arq. Gastroenterol., Jul 2002, vol.39, no.3, p.163-172.

9. AVERBACH, M; BORGES, JLA. Diagnóstico de câncer colorretal. In: ROSSI, BM; et al. Câncer de cólon, reto e ânus. $1^{a}$. Ed. São Paulo: Lemar e Tecmedd Editora, 2004. p. 63 - 75.
10. ALMEIDA FFN e col. - Rastreamento do câncer colorretal. Rev. Hosp. Clín. Fac. Med. S. Paulo 55 (1):35-42, 2000.

11. SCHOTTENFELD, D; WINAWER, SJ. Large intestine. In: SCHOTTENFELD, D; FRAUMENI JR, JF. Cancer epidemiology and prevention. Philadelphia: Saunders, 1982. p. $703-27$.

12. COTTI, G; et al. Tumores colorretais sincrônicos. Coloproct 2006;v. 26; supl.1: p.33.

13. CAMPOS, FG; et al. Tumores colorretais metacrônicos. Coloproct 2006;v. 26; supl.1: p.33.

14. CORDEIRO, F. Diretrizes para diagnóstico, estadiamento e tratamento cirúrgico e multidisciplinar do câncer colorretal. Rev. Assoc. Med. Bras., 2004, vol.50, no.1, p.10-11.

15. MAXFIELD RG - Colonoscopy as a routine preoperative procedure for carcinoma of the colon. Am J Surg 1984; 147: 477-80.

16. GOWEN GF - The impact of colonoscopy on colorectal carcinoma. Am Surg 1991; 57: 254-8.

17. LATIF, J; LEIRO, FO; ALTUNA, F; ARRASCAITE, S; BARREDO, C. Cáncer sincrónico del colon y del recto / Colorectal synchronous câncer. Rev. argent. coloproctología;9(4):158-64, dic. 1998.

18. GOLEMATIS BC, TZARDIS PJ, AHWAL JAL, CHARITOPOULOS N, PEVERETOS P. Site distribution of carcinoma of the large intestine. Retrospective study of 600 cases. Dis Colon Rectum 1989;32:14-6.

19. NAKAO, FS; CURY, MS; FERRARI Jr., AP Colonoscopia ou sigmoidoscopia: risco de lesões isoladas no cólon direito. Arq. Gastroenterol., Set 2001, vol.38, no.3, p.168-171. 
20. ROCHA, BP; FERRARI, AP; FORONES, NM Freqüência de pólipos em doentes operados de câncer colorretal. Arq. Gastroenterol., Jan 2000, vol.37, no.1, p.31-34.

21. FERREIRA, FO; NAKAGAWA, WT. Seguimento de pacientes tratados de câncer colorretal. In: ROSSI, BM; et al. Câncer de cólon, reto e ânus. $1^{a}$. Ed. São Paulo: Lemar e Tecmedd Editora, 2004. p. 553 - 71.

22. MÄKELÄ J, KIVINIEMI H, LAITINEN S. Survival after operations for colorectal cancer in patients aged 75 years or over. Eur J Surg 2000;166:473-9.

23. MULCAHY HE, PATCHETT SE, DALY L, O'Donoghue DP. Prognosis of elderly patients with large bowel cancer. $\mathrm{Br}$ J Surg 1994;81:736-8.
24. ALICI S, AYKAN NF, SAKAR B, BUTUTLAR G, KAYTAN E, TOPUZ E. Colorectal cancer in young patients: characteristics and outcome. Tohoku J Exp Med 2003; 199(2):85-93.

\section{Endereço para correspondência:}

SÉRGIO MURILO DA SILVA ANDRADE

COREME - Hospital Universitário Alcides Carneiro

Rua Carlos Chagas, s/n - Bairro São José

Campina Grande - PB.

E-mail: sergioandrademurilo@hotmail.com 